\title{
Emergencias extrahospitalarias: el paciente suicida
}

\author{
Pacheco Tabuenca T. ${ }^{1}$, Robles Sánchez JI. ${ }^{2}$
}

Sanid. mil. 2011; 67 (4): 345-353; ISSN: 1887-8571

\begin{abstract}
RESUMEN:
Introducción: La conducta suicida ha existido desde que la humanidad existe, sin embargo, la concepción positiva o negativa del mismo ha cambiado a través de las diferentes culturas. En la actualidad, supone un grave problema de salud pública, y se estudia desde diferentes perspectivas y áreas de conocimiento, tratando de dar una visión global para poder entender este fenómeno tan interesante, apasionante y, en ciertos momentos desconcertante. Objetivo: Por tanto, el objetivo del presente estudio es analizar el perfil de las personas que cometen un acto suicida en la ciudad de Madrid y que son atendidos en el área de la emergencia extrahospitalaria. Método: Estudio observacional, descriptivo y retrospectivo, encuadrado en SAMUR-Protección Civil durante el año 2008, analizando todos los casos atendidos de ideación, tentativa y suicidio consumado donde han intervenido un Soporte Vital Avanzado y la Unidad de Asistencia Psicológica. Se ha analizado una muestra de 96 pacientes suicidas, donde el 48,4\% fueron tentativas de suicidio, seguidos de un 44,2\% de suicidios consumados y un 7,4\% de ideación autolítica, atendidos principalmente en los meses de marzo y mayo, turno de mañana - tarde, y en el distrito de Puente de Vallecas, intervenciones demandadas desde Madrid 112 en su mayor porcentaje. Resultados: Se establece un perfil de paciente suicida como varón, de 25-35 años, español, soltero, con patología psiquiátrica en tratamiento, sin tentativas previas, que había verbalizado sus intenciones autolíticas, teniendo como factor desencadenante la propia enfermedad física o psíquica, que elige como método autolítico la defenestración sin presencia de otras personas, al cuál se le traslada al Hospital y presenta como diagnóstico principal TCE, trauma o politraumatismo. Conclusiones: El conocimiento del perfil del paciente suicida atendidos en diversas áreas permite conocer en mayor medida, los factores de riesgo de la conducta suicida, así como puede favorecer actuaciones preventivas futuras.
\end{abstract}

PALABRAS CLAVE: Suicidio, Emergencias, Extrahospitalaria.

\section{Out-of-hospital emergencies: the suicidal patient}

SUMMARY:

Background: Suicidal behavior has existed since mankind exists, however, positive or negative view of it has changed through different cultures. At present, poses a significant public health problem, and studied from different perspectives and areas of expertise, trying to give an overview to understand this phenomenon so interesting, exciting and at times bewildering. Therefore, the objective of this study is to analyze the profile of people who commit a suicide in the city of Madrid and are treated in the outpatient emergency area. Methods: Observational, descriptive and retrospective study, framed in SAMUR-Civil Protection in 2008, analyzing all cases seen ideation, suicide attempt, which have involved an Advanced Life Support and Counselling Unit. We analyzed a sample of 96 suicidal patients, where 48.4\% were attempted suicide, followed by $44.2 \%$ of suicides and suicidal ideation $7.4 \%$, seen mainly in the months of March and May, morning session-afternoon, and in the district of Puente de Vallecas, intervention from Madrid 112 defendants in the highest percentage. Results: Establishing a patient profile suicide as male, 25-35 years, Spanish, single, with psychiatric disorders in treatment, but previous attempts, he had verbalized his intentions autolytic, taking as a trigger for the physical or mental illness itself, which chooses defenestration as autolytic method without the presence of others, to which he was transferred to the Hospital and has a diagnosis of TBI, trauma or polytrauma. Conclusions: Knowledge of the profile of the suicidal patient treated in different areas allows better known, the risk factors of suicidal behavior and may facilitate future preventive actions.

KEY WORDS: Suicide, Emergency, Outpatient.

\section{INTRODUCCIÓN}

El acto suicida ha existido desde que la humanidad existe, sin embargo, la concepción positiva o negativa del mismo ha cambiado a través de las diferentes culturas. En la actualidad, la Organización

\footnotetext{
${ }^{1}$ Psicóloga. Ayuntamiento de Madrid. Subdirección General SAMUR-Protección Civil España.

2 Cte. Psicólogo. Escuela Militar de Sanidad. Departamento Psicología. Madrid. España.
}

Dirección para correspondencia: Teresa Pacheco Tabuenca. Psicóloga de Guardia. SAMURProtección Civil. Ronda de las Provincias s/n. Casa de Campo. 28011 Madrid.

Email: pachecott@madrid.es

Recibido: 28 de junio de 2010

Aceptado: 14 de febrero de 2011
Mundial de la Salud señala el grave problema de salud pública ante el que nos encontramos con la conducta suicida. En su informe sobre la violencia en el mundo, expone que en el año 2000, 815.000 personas habrían fallecido por suicidio en todo el mundo, siendo la decimotercera causa de muerte en el mundo y la cuarta causa en sujetos de entre 15 y 44 años y la sexta de incapacidad ${ }^{1}$.

A través de los datos del World Health Report se estima que en la región Euro A (a la que pertenece España) se produjeron 54.280 muertes por suicidio en el año $2000^{2}$. España presenta una de las tasas de suicidio más bajas $(8,7$ por 100.000$)$, pero ha sufrido, junto a Irlanda, uno de los incrementos en las tasas más altos de Europa y del mundo. En nuestro país se puede observar un aumento en las tasas de suicidio desde 1975 hasta 1994, produciéndose una estabilización 
en los años siguientes; este incremento es más pronunciado entre los varones que entre las mujeres. Los factores sociales, especialmente ligados a los papeles de sexo y los cambios en estos papeles, son las explicaciones más probables. Otra posible explicación del aumento observado de la mortalidad por suicidio entre los varones jóvenes podría ser la epidemia de sida y de adicción a drogas por vía parenteral que se observó en España en los años ochenta y noventa ${ }^{3}$.

Existen dos estudios recientes realizados simultáneamente en varios países europeos. El primero de ellos ${ }^{4}$, señaló una prevalencia vital del 4,4\% para la ideación suicida y de un $1,48 \%$ para los intentos de suicidio en España, mientras que la media europea para la ideación suicida fue del 7,8\% y para los intentos de suicidio del $1,81 \%$. Dentro de la población española el riesgo asociado a los precursores del suicidio estudiados fue significativamente mayor en las mujeres, las cohortes más jóvenes y los niveles educativos menores. La presencia de un trastorno mental se asoció con un aumento del riesgo en todas las categorías diagnósticas estudiadas, especialmente alto en el episodio depresivo mayor. El primer año desde el inicio de la ideación fue el de mayor riesgo de realizar un intento para disminuir a partir de entonces ${ }^{5}$.

Ocurren más muertes por suicidio que la suma de homicidios y guerras, y a nivel global la mortalidad por suicidio es equiparable a la que ocurre por accidentes de tráfico ${ }^{6,7}$. Si comparamos las muertes anuales por suicidio y accidente de tráfico, según datos oficiales en el 2007 fallecieron en España 3263 personas por suicidio ${ }^{8}$, mientras que por accidente de tráfico fallecieron 3811 personas.

Dentro de las primeras atenciones que recibe una persona que comete un acto suicida, son las llevadas a cabo en el ámbito extrahospitalario, siendo el área donde se enclava este estudio, en concreto, dentro del Servicio de Emergencia Extrahospitalaria SAMUR-Protección Civil, perteneciente a la Dirección General de Emergencias y Protección Civil (Ayuntamiento de Madrid), que da respuesta a todos aquellos sucesos acaecidos en vía pública, instituciones y locales públicos de la Ciudad de Madrid.

El objetivo del presente artículo es dar a conocer un estudio epidemiológico que ha permitido conocer la incidencia y las características de los intentos de suicidio y suicidios consumados in situ en la población madrileña (mortalidad, método empleado, tentativas previas, antecedentes psicopatológicos, tratamiento anterior y verbalización previa), con el fin último de conocer factores de riesgo en la conducta suicida y posibilitar con ello actuaciones preventivas.

El tema del suicidio es un problema de interés en la gran mayoría de los ejércitos del mundo, incluido el español. Las últimas noticias de prensa refieren como hay más bajas en el ejército norteamericano por suicidio que las producidas por enfrentamientos armados; a pesar de todo, una sola muerte por suicidio es excesiva. Por ello, es objeto de estudio con la finalidad de introducir medidas preventivas que puedan, si ello fuera posible, eliminar el número de muertes por esta causa o al menos reducirlas al máximo, y aunque los datos del presente estudio son difícilmente extrapolables al ámbito operativo militar, sus resultados no dejan de ser interesantes para las fuerzas armadas.

\section{MÉTODO}

Se trata de un estudio observacional descriptivo transversal, enclavado en el Servicio de Emergencia Extrahospitalaria SAMURProtección Civil en la ciudad de Madrid.
Actualmente el término municipal de Madrid tiene una extensión de $604,20 \mathrm{~km}^{2}$ y una población de derecho de 3.213.271 habitantes, además de aquellas personas que por diferentes motivos acuden a la capital. Los equipos del SAMUR-Protección Civil, compuestos por médicos, psicólogos, enfermeros y técnicos en emergencias, han atendido en el año 2008 un total de 124.722 emergencias.

La muestra total de estudio fueron 96 sujetos, atendiendo a los siguientes criterios de inclusión: sujetos atendidos durante el 2008 por un suceso codificado inicialmente como posible ideación, tentativa o suicidio consumado, haber sido atendido por un Soporte Vital Avanzado, existir una activación del psicólogo para dicho suceso, que en el informe de asistencia viniera reflejado que era ideación suicida, tentativa de suicidio o suicidio consumado, eliminando los que se confirma in situ que son accidente laboral.

Para evaluar las variables necesarias se emplearon varios instrumentos. En primer lugar se revisó la Base de Datos de Asistencias de SAMUR-Protección Civil, en segundo lugar los Informes de Asistencia de Soporte Vital Avanzado (SVA) e Informes de Asistencia de Intervención Psicológica.

Para el análisis de los datos se clasificaron las variables de estudio atendiendo a las características del suceso e intervención (mes, horario, distrito, demandante, destino, hospital, código Inicial y final SVA, familia u otros presentes durante acto suicida y diagnóstico CIE 9); características sociodemográficas (género, edad, nacionalidad, país y estado civil) y características clínicas del paciente suicida (anteriores intentos autolíticos, método anterior, verbalización anterior de ideación autolítica, diagnóstico previo, tratamiento psiquiátrico o psicológico, factor desencadenante, método empleado).

De acuerdo con la Ley Orgánica de Protección de Datos vigente, toda la información recogida fue tratada con fines estadísticos de manera global y confidencial a nivel individual.

El diseño de estudio cuenta con la limitación que introduce el manejar documentación ya elaborada, con datos recogidos por parte del profesional que atiende. Podemos encontrar por tanto, con un sesgo de información por la falta de precisión que aparece cuando se recurre a la historia clínica o psicológica.

Para el tratamiento estadístico de los datos se ha utilizado el paquete estadístico SPSS para Windows (versión 15.0), con licencia de uso para SAMUR-Protección Civil. Se ha utilizado la distribución de frecuencias para analizar la prevalencia de las características del suceso e intervención, las características sociodemográficas y las características clínicas de la población estudiada. Para analizar las diferencias entre variables se ha utilizado como prueba estadística la prueba t, F o Chi cuadrado, atendiendo al tipo de variables.

\section{RESULTADOS}

\section{Características del Suceso e Intervención}

Dentro de la muestra total de 96 pacientes que han realizado un acto suicida, el $48,4 \%$ fueron tentativas de suicidio, seguidos de un $44,2 \%$ de suicidios consumados y un 7,4\% de ideación autolítica.

En cuanto a la variable mes, los sucesos tuvieron lugar en un $12,1 \%$ en los meses de Marzo y Mayo, seguidos por un 11,1\% en los meses de Enero, Junio y Septiembre, 9,1\% Febrero, Abril, y un porcentaje menor o igual a $7,1 \%$ el resto de los meses (Figura 1). 


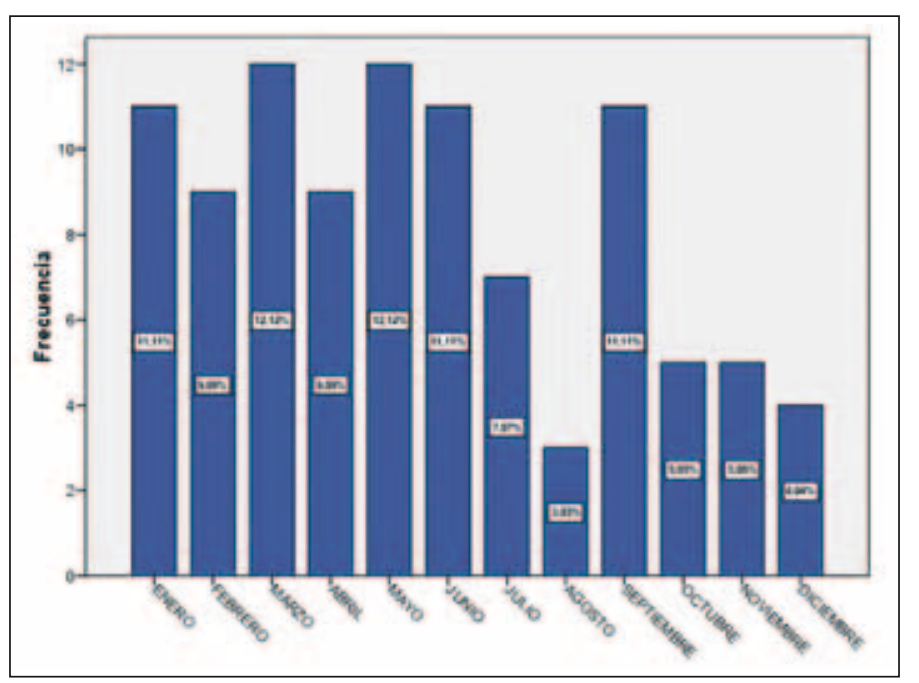

Figura 1. Actos suicidas atendidos por mes.

El momento del día en que fueron atendidos fue en un 39,4\% en turnos de mañana y tarde, seguidos de $21,2 \%$ en turno de noche.

El 11,2\% de los pacientes fueron atendidos en el distrito de Puente de Vallecas, 7,1\% en los distritos de Centro, Moncloa-Aravaca y Carabanchel, 6,1\% Usera y Latina, 5,1\% Salamanca, Fuencarral-El Pardo, Ciudad Lineal y Hortaleza, 4,1\% Chamartín, Villaverde y Villa de Vallecas, 3,1\% Arganzuela, Retiro, Chamberí y Moratalaz, 2\% Tetuán y Vicálvaro, $1 \%$ Barajas.

El 72,4\% de los sucesos fueron demandados por Madrid 112, 12,2\% por Policía Nacional, 6,1\% por Policía Municipal, 3,1\% por SUMMA e Insalud, $2 \%$ por Agentes de Movilidad y $1 \%$ por Bomberos.

El $48,5 \%$ de los pacientes fueron trasladados a hospital, en el $38,4 \%$ de los casos se certificó el fallecimiento in situ, en el $4,0 \%$ se derivó a otro recurso y en el 9,1\% el destino fue otro (trasladado por familiares, etc.).

Respecto a los hospitales donde fueron traslados los pacientes, el 11,2\% fue al Hospital Clínico, 7,1\% al Gregorio Marañón, 6,1\% Doce de Octubre, La Paz y Ramón y Cajal, 4,1\% al Puerta de Hierro y $2 \%$ a La Princesa y La Concepción (Figura 2).

La codificación final de la atención realizada por SVA fue en el $65,6 \%$ defenestración, $12,9 \%$ autolisis traumática, 5,4\% cadáver en

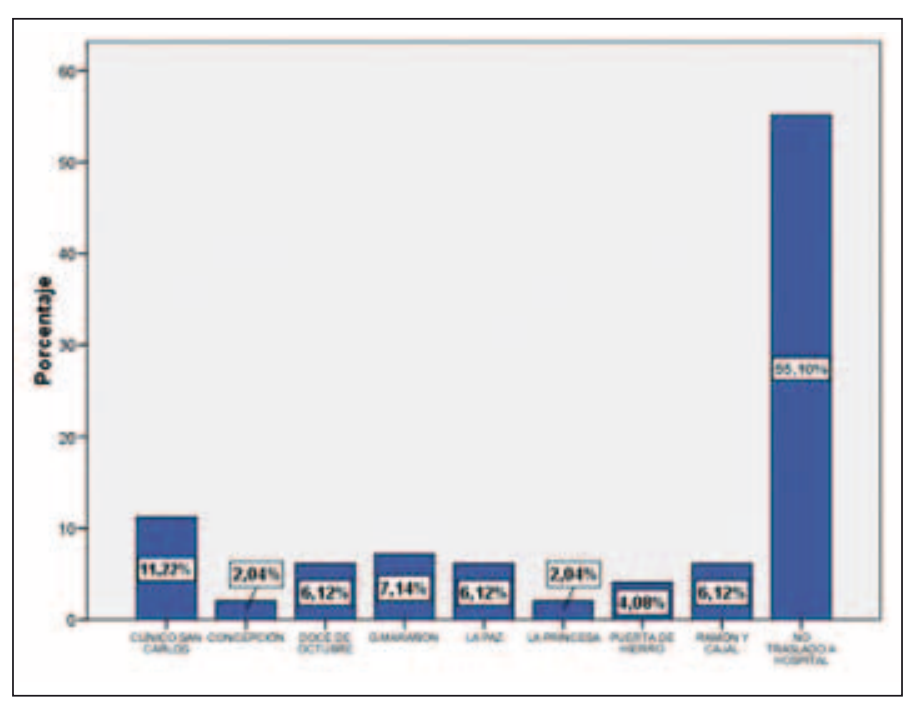

Figura 2. Hospitales de destino. vía pública, 4,3\% arrollado tren y certificación psiquiátrica, 3,2\% cadáver en domicilio, $1,1 \%$ arma de fuego, sobredosis, intoxicación por fármacos y/o tóxicos y autolisis no traumática.

El diagnóstico final realizado por el facultativo del SVA atendiendo a la codificación CIE-9 fue en un 48,4\% éxitus, 24,7\% TCE, trauma o politrauma, $9,7 \%$ patología psiquiátrica, $8,6 \%$ posible fractura, 3,2\% herida abierta o complicada, 2,2\% intoxicación por fármacos o tóxicos, $1,1 \%$ contusiones, policontusiones, esguince $\mathrm{o}$ luxación, amputación o síndrome de aplastamiento (Figura 3).

En el lugar del suceso y en el mismo momento de llevar a cabo la conducta suicida, en un $49,2 \%$ de los casos había estado alguna otra persona presente (familiar, allegado o testigo) y en un $50,8 \%$ no había nadie.

\section{Características Sociodemográficas de la Población Suicida}

De la muestra total de pacientes atendidos el 60,4\% eran hombres y el 39,6\% eran mujeres. Con edades comprendidas entre los 15 y 92 años, el 1,1\% eran entre $0-15$ años, $11,8 \%$ de $16-24,25,4 \%$ de $25-35,21,2 \%$ de $36-45,11,7 \%$ de $46-55,17 \%$ de 56 a $65,4,3 \%$ de $66-75,8,8 \%$ mayor de 76 , siendo la media de edad 44,98 (desviación típica de 18,5) (Figura 4).

En cuanto a la nacionalidad de esta población, el $82,3 \%$ eran españoles, $16,7 \%$ extranjeros y en un $1 \%$ no constaba la nacionalidad. El 47,4\% eran solteros, el 23,2\% eran casados, $4,2 \%$ viudos o en convivencia marital, $3,2 \%$ separados o divorciados, y en un $17,9 \%$ de los casos no se reflejaba en el informe el estado civil de la persona.

\section{Características Clínicas de la Población Suicida}

En el 46,7\% de los casos se recogió que el paciente no había tenido anteriores tentativas suicidas, en el $25 \%$ si que las había tenido y en el $28,3 \%$ de los casos no se recogía esta información. En cuanto al método empleado en el caso de anteriores tentativas suicidas, los sujetos había utilizado en el 60,95 de los casos la intoxicación con fármacos sedantes, en el 17,4\% la defenestración, en el 8,7\% la in-

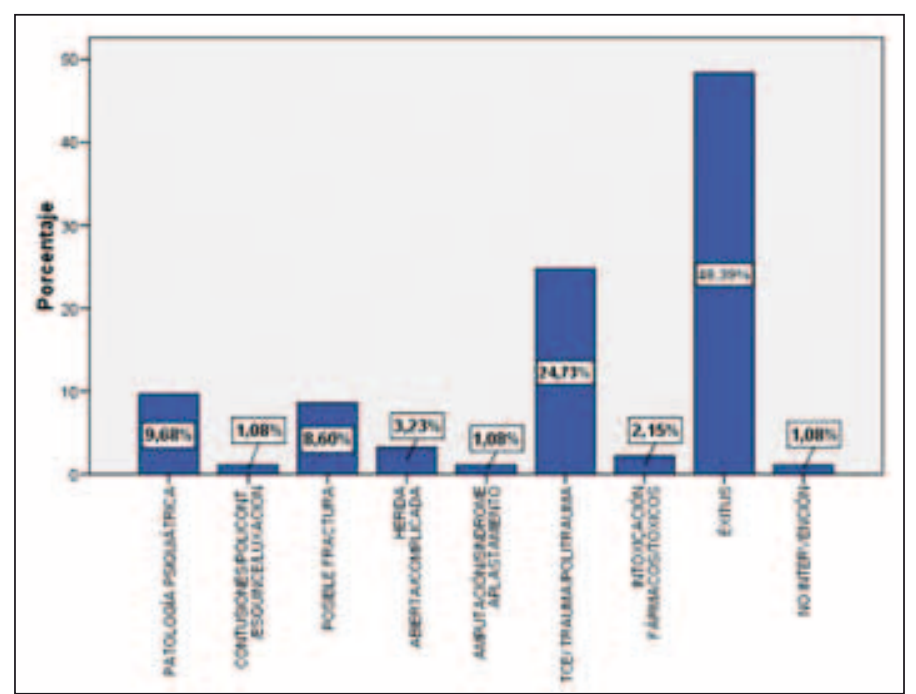

Figura 3. Diagnóstico CIE-9 tras suceso. 


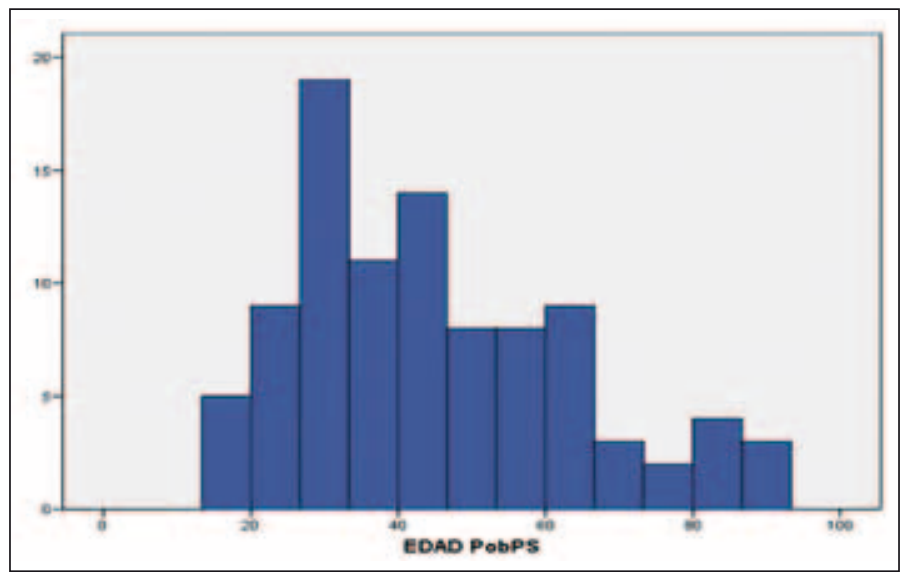

Figura 4. Distribución Edad PobPS.

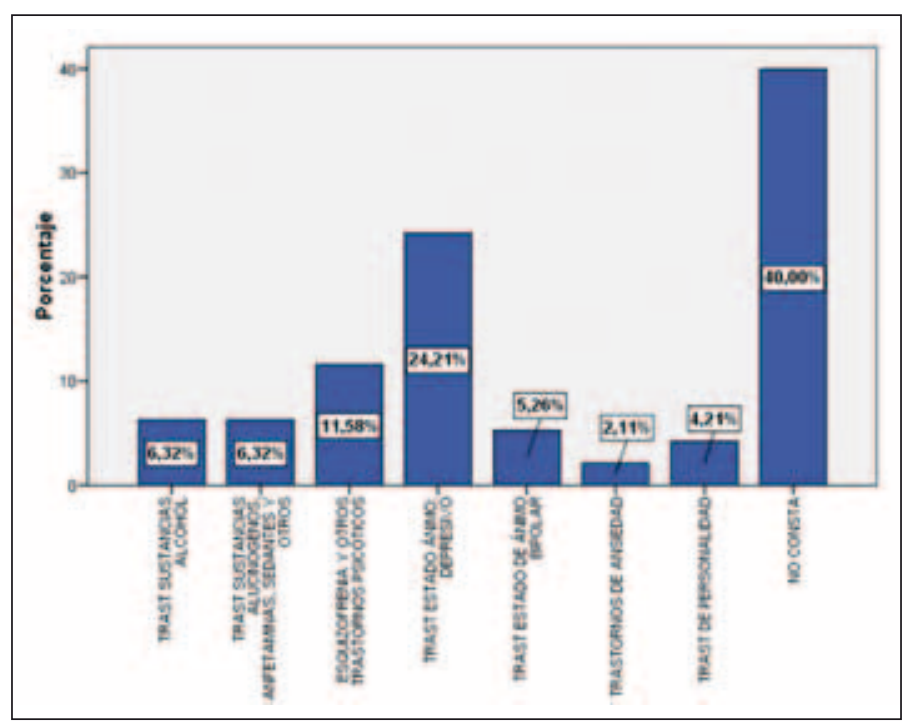

Figura 5. Diagnóstico previo PobPS.

toxicación con fármacos no sedantes u otros, en el 4,3\% con arma de fuego y en el 8,7\% no constaba el método empleado (Figura 5).

En el acto suicida actual el método empleado en el 76,8\% de los casos fue la defenestración, seguido de un 9,5\% el ahorcamiento, $5,3 \%$ arma de fuego, $4,2 \%$ arrojarse al metro o tren y $1,1 \%$ empleando la intoxicación por fármacos con efecto sedante, cortes o heridas incisivas, intoxicación por gas y método mixto (Figura 6).

El $47,1 \%$ había verbalizado anteriormente sus intenciones de cometer el acto suicida y en el $22,4 \%$ no. En un $30,6 \%$ no constaba si lo había verbalizado o no. De los pacientes atendidos, el $60 \%$ de los sujetos tenían un diagnóstico psiquiátrico previo, mientras que en el $40 \%$ de los casos no constaba en el informe. De los sujetos que tenían diagnóstico previo el $24,2 \%$ era de Trastorno del estado del ánimo Depresivo, el 12,6\% Trastornos por consumo de sustancias, el 11,6\% Esquizofrenia y otros trastornos psicóticos, el 5,3\% Trastorno del estado de ánimo Bipolar, 4,2\% Trastorno de Personalidad y $2,1 \%$ Trastorno de Ansiedad (Figura 7).

El 44, 7\% estaba en tratamiento en la actualidad, el $23,4 \%$ sin tratamiento y encontrando en $31,9 \%$ de los casos donde no contaba información al respecto. En cuanto al tipo de tratamiento, el $74 \%$ recibían tratamiento psiquiátrico o psicológico, en el 14\% recibían tratamiento por parte del médico de cabecera y en un $12 \%$ no consta.

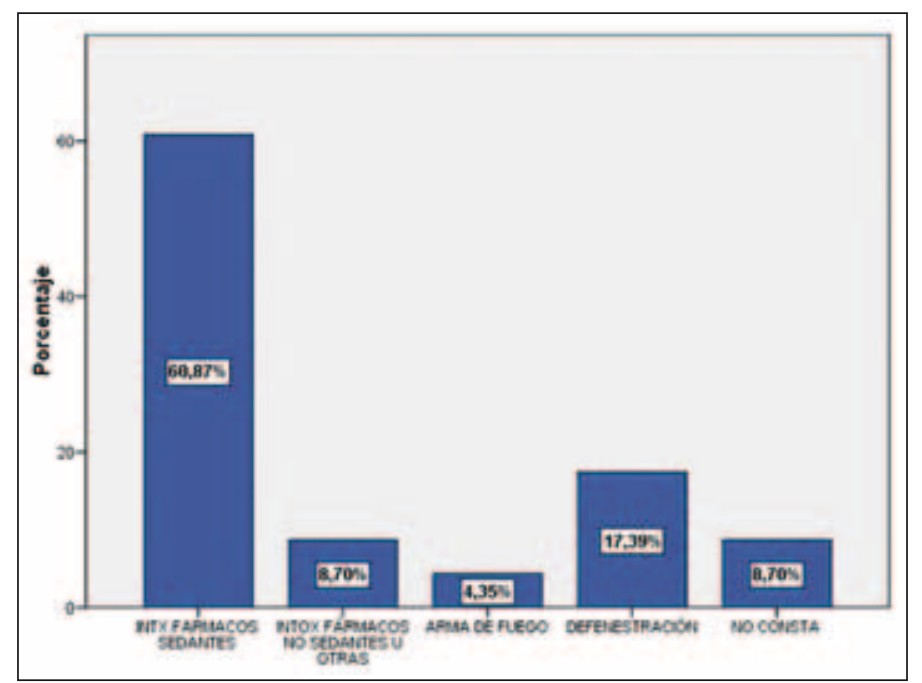

Figura 6. Método empleado suceso actual.

Respecto al factor desencadenante que motivó la conducta suicida en el 43,55 de los casos fue por enfermedad (física o psíquica), en el 4,3\% por acontecimientos vitales estresantes, problemas familiares, escolares, laborales, intoxicación aguda en el momento, y en el $1,1 \%$ por otros motivos, no constando el desencadenante en el $38 \%$.

\section{Análisis de la población suicida atendiendo a las variables demográficas}

En primer lugar, se analizó la PobPS atendiendo a las variables demográficas género y edad. Al comparar la puntuación media en la PobPS atendiendo a las variables demográficas de género y edad, donde la media de edad de los varones era 47,17 (desviación típica de 17,660$)$ frente a la media de edad de las mujeres que era inferior (41,54, desviación típica 19,551) (Figura 8). No se obtuvieron diferencias significativas $(\mathrm{t}=1,454, \mathrm{p}>0,05)$.

Posteriormente se analizó como se distribuía la PobPS en la variable género dentro de cada tipo de conducta suicida (Figura 9), obteniéndose que los varones presentaban ideación autolítica en un

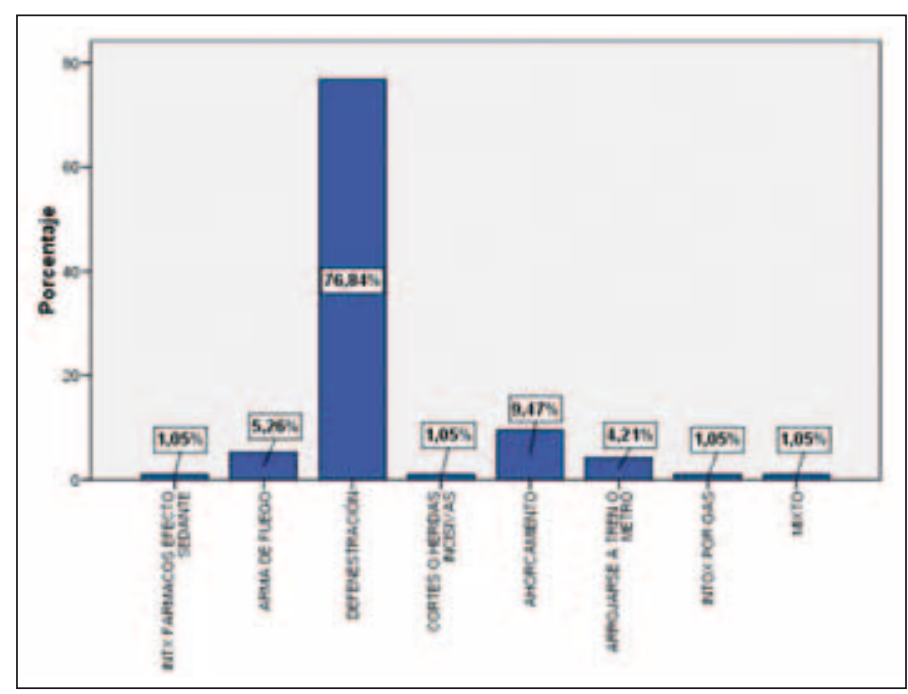

Figura 7. Método empleado tentativas anteriores. 


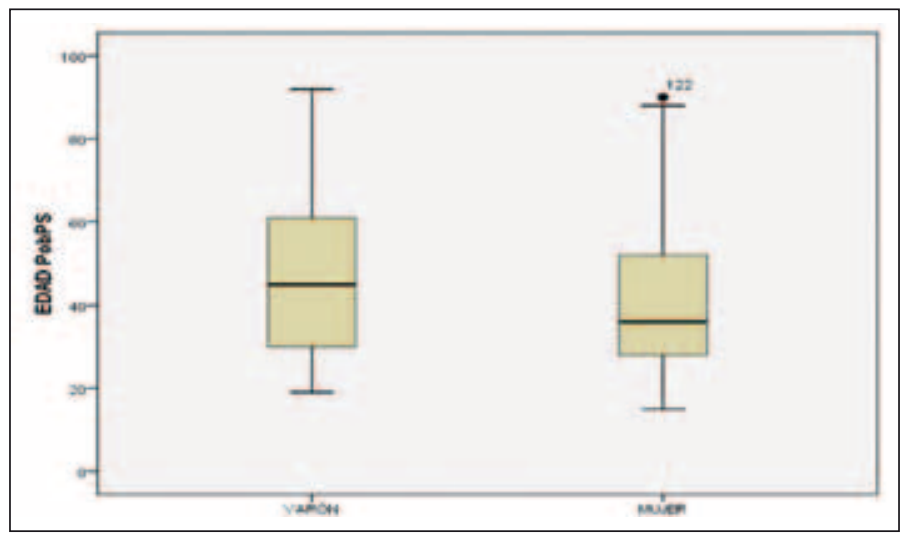

Figura 8. Distribución Edad-Género PobPS.

$71,4 \%$ frente a las mujeres en un $28,6 \%$; la tentativa de suicidio era realizada por los varones en un $52,2 \%$ frente a un $47,8 \%$ de las mujeres, y el suicidio consumado era llevado a cabo por varones en un $66,7 \%$ frente al $33,3 \%$ de las mujeres. Para conocer la relación entre ambas variables se realizó la prueba de independencia Chi-cuadrado, obteniéndose un nivel de significación mayor a $0.05(\mathrm{p}=0,312)$.

En cuanto a la distribución de la edad de la Pob PS en cada uno de los tipos de conducta suicida, la ideación autolítica tenía una media de edad de 47,0 (desviación típica 22, 325) con una edad mínima de 28 y máxima de 83 años; la tentativa suicida tenía una edad media de 39,50 (desviación típica de 16,339) con una edad mínima de 15 y máxima de 88 años; el suicidio consumado presentaba una edad media de 51,05 (desviación típica 18,852) con una edad mínima de 20 años y edad máxima de 92 años. Tras el análisis de la relación entre ambas variables se obtuvo que existían diferencias significativas intergrupos $(\mathrm{F}=4,616, \mathrm{p}=0,012)$, obteniéndose con la prueba post hoc de Bonferroni una $\mathrm{p}=0,010$ entre tentativa de suicidio y suicidio consumado.

\section{Análisis de pacientes suicidas atendiendo a las características del suceso}

En el análisis de la variable distrito, encontramos que existen diferencias significativas atendiendo a la verbalización anterior del paciente suicida de su intención suicida $(F=2,204, p=0,010)$, encontrando que distritos como Chamberí, Moncloa-Aravaca y Vicalvaro tienen mayor incidencia de sujetos que verbalizan antes la ideación o intención suicida (Figura 10).

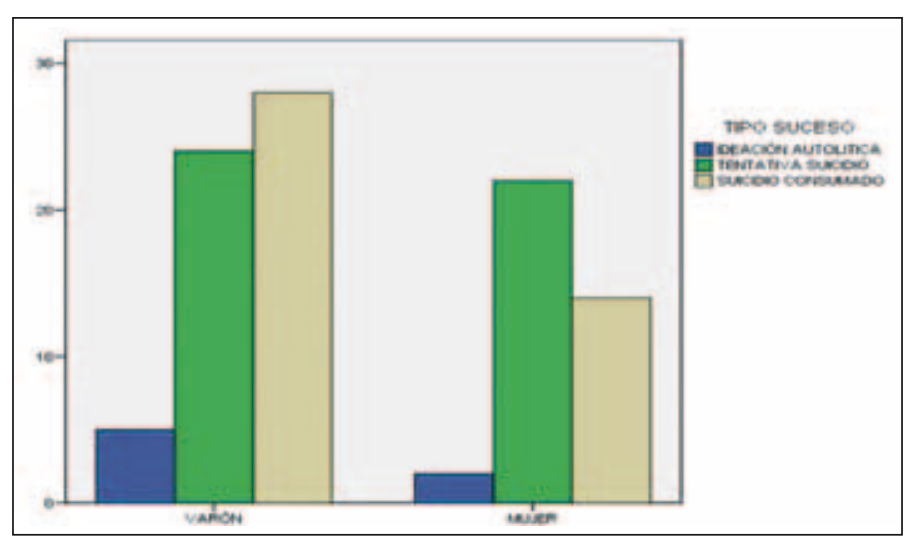

Figura 9. Distribución de la variable género en tipo de suceso.

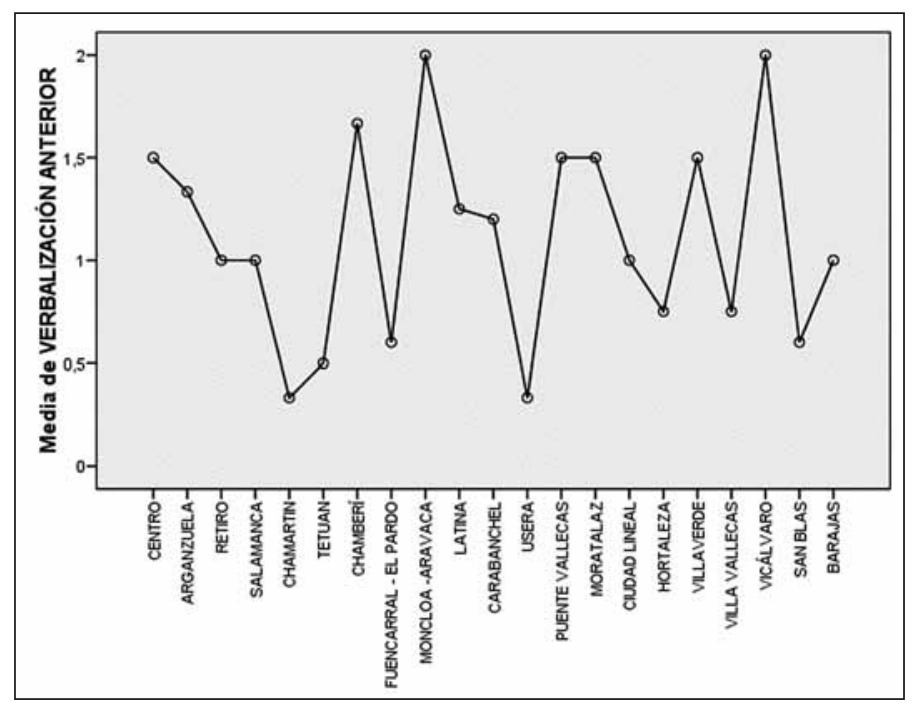

Figura 10. Verbalización ideación suicida por distrito.

$\mathrm{Al}$ analizar el turno en el que se produce el suceso, existen diferencias significativas en cuanto hospital al que se traslada $(\mathrm{F}=2,984$, $\mathrm{p}=0,055$ ). Al estudiar la variable tipo de suceso (ideación, tentativa o suicidio consumado) encontramos que existen diferencias atendiendo al destino del paciente suicida con $\mathrm{F}=19,129$ y $\mathrm{p}=0$ y el factor desencadenante de la conducta suicida con una $F=5,039$ y $\mathrm{p}=0,008$. Además se encuentran diferencias significativas en cuanto a la realización de comunicación de malas noticias con $\mathrm{F}=69,053$ y $\mathrm{p}=0$. Así mismo, se encuentran diferencias significativas en cuanto al diagnóstico final del paciente suicida (Código Patológico SVA) con $\mathrm{F}=33,225 \mathrm{y}$ p=0, en función de los diferentes tipos de suceso. Así mismo, se encontraron diferencias en cuanto a la presencia de familiares o allegados en el lugar mientras se cometía el acto suicida en función del método $(\mathrm{F}=2,731, \mathrm{p}=0,013)$, con mayor número de presencia de familia o allegados cuando el método es mediante cortes o heridas incisivas o mixto (Figura 11).

\section{DISCUSIÓN}

Los resultados obtenidos señalan que hay un $48,4 \%$ de tentativas de suicidio. Coincide con los datos aportados por Mingote et al. (2004) que reflejan que por cada persona que fallece por suicidio otras 22 acuden a los servicios de urgencias por conductas suicidas,

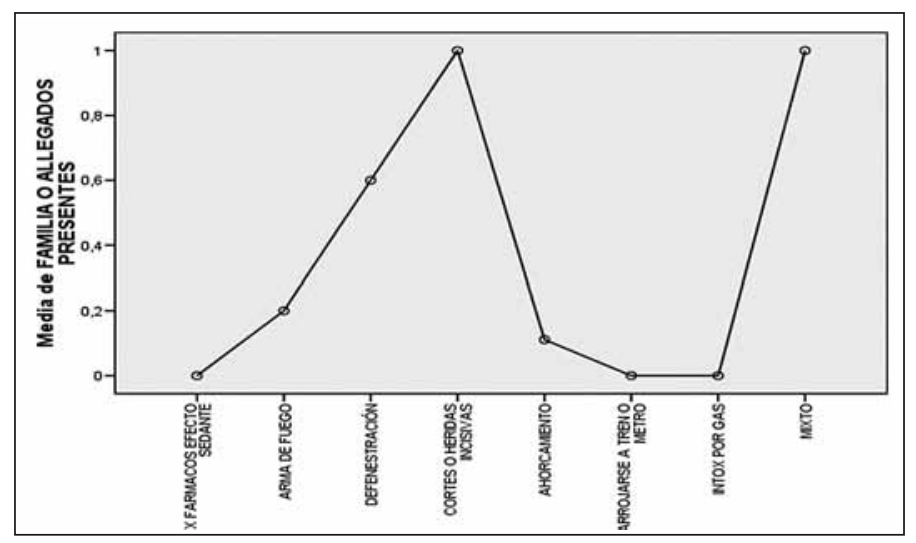

Figura 11. Familia u otros presentes atendiendo al método suicida. 
estimando que en Europa suponen cerca de un millón de urgencias hospitalarias, estimando que la conducta suicida está presente en el $38 \%$ de las urgencias psiquiátricas ${ }^{9}$. Mateos et al. (2007) en su estudio dentro del ámbito extrahospitalario señalan que casi un $1 \%$ del millón de las llamadas que recibe un Servicio de Emergencias en la Comunicad de Madrid son por un intento autolítico donde se había empleado fármacos o tóxicos ${ }^{10}$. En un estudio realizado en la Comunidad de Madrid, se estima una incidencia anual de 102,07 tentativas autolíticas por cien mil habitantes ${ }^{11}$. Destaca la diferencia con los datos oficiales de tentativas de suicidio en España, confirmando así la variabilidad de datos y la inexistencia de datos fiables respecto a este hecho. También es importante hacer referencia a los datos facilitados en diferentes estudios y que ha sido expuestos anteriormente, donde se habla de una mayor incidencia de ideación suicida que intentos de suicidio o suicidio consumado, ya que dependiendo del ámbito donde se estudie, estos datos varían. Un servicio de emergencias se solicitará principalmente cuando la conducta ya se ha realizado y en pocos casos como podemos observar en la muestra estudiada, se solicitará cuando simplemente se está desarrollando la idea.

En cuanto al momento estacional en que se producen los actos suicidas, los resultados señalan que los meses de Enero, Marzo, Mayo, Junio y Septiembre presentan mayor incidencia, datos que coinciden con los datos oficiales de España en el 2007 donde se encuentra que los meses de mayor incidencia son Marzo, Junio y Septiembre. El suicidio ha sido relacionado con la temperatura, humedad, vientos y fase lunar, existiendo resultados contradictorios. Durkheim señala que el factor climático no influye en la conducta suicida, sin embargo, encontró una mayor frecuencia de suicidios en primavera y verano ${ }^{12}$.

Las intervenciones se realizan en el mismo porcentaje por la mañana y la tarde $(39,4 \%)$. Coinciden con los datos encontrados en una revisión de 164 tentativas autolíticas mediante fármacos y /o tóxi$\cos$ atendidas en un servicio de emergencia prehospitalaria, donde el 51,8\% lo realizaron de día (07:01-20:59 h) y el 48,2\% de noche (21:00-07:00 h), siendo las mujeres las que más frecuentemente lo realizan y de día (13). El momento del día en que se solicita un servicio de emergencias puede venir determinado porque el servicio donde se enmarca el estudio, da respuesta en vía pública y/o locales públicos, y en el tramo horario de mañana y tarde, las personas habitualmente están fuera de su domicilio.

Respecto a la zona donde hay una mayor incidencia de sucesos con las características estudiadas los resultados reflejan que el mayor porcentaje de pacientes fueron atendidos en los distritos de Puente de Vallecas, Centro, Moncloa y Carabanchel, todos ellos pertenecientes a la mitad sur de Madrid. No se poseen datos comparativos al respecto, sin embargo, podríamos pensar que este dato puede explicarse con la relación anteriormente citada entre nivel socio-económico, precariedad laboral y vulnerabilidad para desarrollar enfermedades mentales. No obstante, deberían tenerse datos para poder concluir esto.

Dentro del Servicio donde se enclava el estudio el mayor porcentaje de las atenciones son demandadas a través de Madrid 112. Los resultados señalan que el $72,4 \%$ fueron demandados por Madrid 112 , es decir a través de las llamadas que todo ciudadano de Madrid realiza por esa vía.

En cuanto a la finalización de las intervenciones con los pacientes suicidas, encontramos que el mayor porcentaje, (48,5\%) fueron trasladados a un hospital. Coincide con los datos de que el mayor número de casos atendidos son tentativas de suicidio, dado que si fueran suicidios consumados no se realizaría traslado. Además, hay que tener en cuenta que cualquier persona con ideación o tentativa suicida siempre va a ser trasladado para valoración psiquiátrica.

Respecto a la codificación inicial de los sucesos, los datos señalan que el 72,3\% de los códigos iniciales de SVA o bien, el motivo de intervención es defenestración como tentativa o suicidio consumado. En cuanto a la patología o lesión producida en los casos atendidos tras la conducta suicida los resultados señalan que el diagnóstico final realizado por el facultativo es de patologías o lesiones de mayor o menor gravedad en un 51,6\% frente a éxitus. Coincide con datos aportados por Torres et al. (2005) que en una muestra de 192 pacientes precipitados desde altura atendidos en emergencia extrahospitalaria se encontró que 54 pacientes críticos presentan politraumatismo $(48,1 \%)$, traumatismo cráneo encefálico $(24,1 \%)$, traumatismo medular $(7,4 \%)$ y traumatismo ortopédico $(5,6 \%)$ frente a $27,8 \%$ de éxitus ${ }^{14}$. La OMS ha calculado como se expuso anteriormente que existen entre 10 y 20 tentativas de suicidio fallidas con el resultado de lesiones, hospitalizaciones, secuelas emocionales y físicas.

Respecto a la presencia de personas mientras el paciente suicida comete el acto, encontramos que en un 50,8\% no había presentes familiares, amigos o testigos presentes en el mismo lugar donde se cometió el acto suicida. Sarró y De la Cruz (1991) señalan que el lugar elegido para consumar el suicidio suele ser el propio domicilio o un lugar frecuentado por el sujeto ${ }^{15}$ y Ayuso (2009) obtiene en su estudio un $88,3 \%$ de sujetos que cometen la tentativa suicida en una ubicación familiar, así como que la persona que indica el rescate tras una tentativa suicida es en un $94,7 \%$ una persona clave, familiar o el propio paciente ${ }^{11}$.

En referencia a las características sociodemográficas del paciente suicida, encontramos que existe un mayor porcentaje de hombres que de mujeres que realizan un acto suicida. Los resultados señalan que de la muestra atendida el $60,4 \%$ son varones. Coincide con los datos aportados por diferentes autores que señalan que la conducta suicida entre varones es por término medio, tres veces superior a la de las mujeres ${ }^{12,16,17,18}$. Por otro lado, los datos aportados reflejan que los varones presentaban mayor incidencia en todos los tipos de actos suicidas, presentando ideación autolítica en un 71,4\%, la tentativa de suicidio con un $52,2 \%$ y el suicidio consumado era realizado por varones en un $66,7 \%$. Los resultados de esta muestra coinciden en primer lugar con la revisión realizada de la conducta suicida en la población española durante el periodo 2004-2006 cuyos datos se reflejaron en el primer apartado de este artículo. Coinciden con los datos que aportan Hintikka et al. (2001) donde la incidencia de la ideación suicida es superior en varones ${ }^{19}$. Respecto a la incidencia de suicidio consumado y tentativa, Montalvo et al. (2009) reflejan los datos con 350 casos de tentativas de suicidio y 25 muertes por suicidio con predominio de varones en los casos de suicidio consumado $(72 \%)$ frente a los casos de intentos de suicidio $(33 \%)^{20}$. Así mismo, las estadísticas muestran claramente cómo en todos los continentes y áreas geográficas existe una mayor incidencia de suicidio entre los varones, estimándose un ratio varón /mujer de 4,1 para el continente americano, en 1,3 en Asia y en 3,0 en Europa. Bobes et al. (2004) señalan que las tasas de parasuicidio y tentativas de suicidio varían considerablemente a tenor de las fuentes de datos consultadas $^{21}$. Sin embargo, a pesar de las diferencias entre los distintos estudios, la gran mayoría de ellos presentan una clara preponderancia 
de la mujer con ratios mujer/varón que en Europa varían entre 1,4 y 4,0 según los países. El estudio WHO/EURO Multicentre Project on Parasuicide que se presentó en 1996 aporta datos que van en esta línea, sin embargo, los autores hacen notar que en algunas áreas las diferencias entre varones y mujeres fueron poco pronunciadas y que existió el caso de Helsinki donde la tasa de tentativas de suicidio fue mayor para varones que para mujeres.

Respecto a la variable edad, los resultados que refleja la muestra estudiada son que los rangos de edad donde se producen con mayor frecuencia actos suicidas son en $25-35$ años $(25,4 \%)$ y $36-$ 45 (21,2\%). Diekstra (1993) expone que existen diferencias entre países, observándose que en algunos hay un primer pico en sujetos jóvenes de 24 a 35 años, y en otros las tasas aumentan hasta las edades medias de la vida ${ }^{16}$. En segundo lugar, se ha obtenido que la edad media de ideación autolítica es de 47, tentativa suicida de 39,5 y suicidio consumado de 51,05. Los datos de edad media en tentativa suicida coinciden con los aportados por Ayuso (2009) que en su muestra obtiene una edad media de 37,17 años ${ }^{11}$. En la misma línea encontramos los resultados al revisar la conducta autolítica en España durante los años 2004-2006, donde la tentativa suicida se da entre los 20-49 años, con un porcentaje superior en el rango 3039 , frente al suicidio consumado que se da en edades superiores, principalmente desde los 30 años, con una frecuencia muy elevada desde los 60 años ${ }^{24}$. Según los datos de la OMS (2002), las personas menores de 45 años representan en la actualidad, más de la mitad de los suicidios que ocurren en un año ${ }^{1}$.

En nuestra muestra, en la variable género encontramos los varones tienen una edad media ligeramente mayor $(47,17)$ que las mujeres $(41,54)$, sin encontrar diferencias estadísticamente significativas. Dentro del estudio realizado con población de Madrid, los datos encontrados reflejan dentro de las tentativas suicidas que la media de edad de varones es 38,5 frente a la edad de las mujeres de 36,5 años ${ }^{11}$.

Respecto a la variable nacionalidad de los sujetos atendidos, el $82,3 \%$ eran españoles. Coincide con los datos que aportan Quintanilla et al. (2008) en su estudio con 239 pacientes que acudieron a urgencias tras una conducta autolesivas ${ }^{22}$, donde sólo el 17,5\% era población inmigrante y con los aportados posteriormente por Ayuso (2009), el cuál obtiene un 13,9\% de población inmigrante en tentativas suicidas ${ }^{11}$.

El análisis de la variable estado civil confirma los datos encontrados en numerosas publicaciones, el acto suicida en nuestra muestra se produce con mayor frecuencia en personas que en el momento del suceso no tienen pareja. Encontramos un 54,8\% de sujetos solteros, viudos, separados o divorciados. En el estudio WHO /EURO se observó en la zona del Norte de Europa una mayor incidencia en mujeres y hombres divorciados y más bajas en viudas y viudos. Entre las mujeres el suicidio alcanza mayores tasas en las divorciadas que entre las casadas. En España, el Instituto Nacional de Estadística en el 1994 publica datos sobre el grado de consumación, sexo y estado civil, obteniéndose que de un total de 1765 suicidios realizados por varones, y donde se recogió el estado civil, 845 estaban solteros, viudos, separados o divorciados frente a 744 casados; en cuanto a los 632 suicidios realizado por mujeres, 271 estaban solteras, viudas, separadas o divorciadas, frente a 252 casadas. Davidson y Philippe (1986) describen un aumento del riesgo de suicidio de 2,3 en hombres solteros, 2,9 en hombres divorciados y 3,6 en viudos, frente a aquellos que se encuentran casados. Asimismo, en las mujeres describen un aumento del riesgo de morir por suicidio de 1,9 en solteras, 2,0 en divorciadas y 2,2 en viudas, frente a las mujeres casadas ${ }^{23}$. Rodríguez et al. (2004), en una muestra de 365 sujetos con conducta suicida, encontraron que hubo predominio en las personas sin vínculo de pareja, divorciados y solteros, para el $67,7 \%$ de la muestra y el 30,4 y $35,1 \%$ respectivamente, seguido de los casados, con el 19,7\% (25). Más recientemente, se obtiene en una muestra de 921 personas que tuvieron una o más tentativas de suicidio, que el $43,1 \%$ estaban solteros, $15,1 \%$ separados o divorciados, $3,5 \%$ viudos frente a un $27,8 \%$ casados $^{11}$. Por otro lado, Harris (1981) al comparar fallecidos por muerte natural con los fallecidos por suicidio, encontró en estos últimos un problema de aislamiento social creciente en los últimos momentos de su vida ${ }^{26}$.

En tercer lugar, se analizan las características clínicas de la población de pacientes suicidas, encontrando que el 46,7 \% no había tenido tentativas anteriores, sin embargo, existe un $28,3 \%$ de casos donde no consta esta información. Estos datos se pueden explicar por varios motivos, siendo uno de ellos, que quizás un porcentaje amplio del que no consta información si que presentaran tentativas anteriores o bien, que podamos analizar la población estudiada como un grupo de riesgo, donde en un porcentaje elevado es su primera tentativa autolítica. Como ya sabemos, la tentativa de suicidio es el principal factor predictor del suicidio consumado, estimando que entre el $10-15 \%$ de los sujetos que realizan una tentativa, acabaran consumando el suicidio, siendo el riesgo mayor durante el primer año posterior a la tentativa. De la misma forma, el 14-60\% de las tentativas tienen antecedentes de una o más tentativas previas y del $30-60 \%$ de los suicidios consumados tienen tentativas previas. Además, se obtiene que existe un porcentaje mayor de sujetos con tentativas anteriores donde habían empleado un método de baja letalidad. Los resultados encontrados son que en un $60,9 \%$ los sujetos habían empleado en tentativas anteriores la intoxicación con fármacos sedantes. Coincide con lo expuesto por Sarro y De la Cruz (1991), donde el método empleado difiere de ser suicidio consumado (más violentos) y tentativas de suicidio (métodos blandos), destacando en estos últimos la intoxicación medicamentosa ${ }^{15}$. En cuanto al método actual empleado, existe un mayor porcentaje de conductas suicidas cuyo método empleado es la precipitación. Los resultados expuestos al respecto nos muestran que el $76,8 \%$ de los casos atendidos emplean como método de suicidio la defenestración. En España, donde el acceso a las armas es más difícil que en otros países como EEUU, los medios más frecuentemente empleados para consumar el suicidio son la precipitación, intoxicación y ahorcamiento. Diversos estudios confirman esos datos, principalmente en zonas urbanas donde la precipitación es el método más utilizado $27,28,29,30$. La OMS en el 2008 publica un mapa de los métodos de suicidio empleados a nivel internacional y concluye que el perfil de los métodos empleados depende de la disponibilidad de los métodos y en concreto de la disponibilidad de medios técnicos. De ahí que señalen la importancia de que se debe restringir el acceso a ciertos medios de suicidio (armas, fármacos, etc. $)^{31}$.

Respecto al momento previo a la conducta suicida, los resultados indican que en la muestra estudiada el 47,1\% había verbalizado anteriormente sus intenciones. Tanto para la familia como para los propios profesionales, estos datos pueden producir y producen una sensación de fracaso de un tratamiento, por cuanto se trata de una conducta que en algunos casos podría prevenirse. Nuestros datos 
coinciden con los obtenidos por otros autores que afirman que más de la mitad de los sujetos que se suicidan habían consultado con el médico en el mes previo a su muerte ${ }^{32}$ y más de un tercio, con un servicio de psiquiatría, en el año anterior ${ }^{33}$. Montalvo et al. (2009) en su estudio encontraron que más del $50 \%$ de los pacientes con tentativa de suicidio habían visitado a su médico de referencia en los últimos 3 meses $^{20}$.

Además, analizando la idea de que los sujetos que se suicidan presentaban un diagnóstico de enfermedad mental previa, en concreto en nuestra muestra se obtiene que en un $60 \%$ de los pacientes suicidas había un diagnóstico psiquiátrico anterior. Al respecto se encuentran numerosos estudios que coinciden con estos datos y de los que ya se ha hecho referencia anteriormente ${ }^{5,11,34}$, así como aquellos que señalan que la existencia de una alteración psiquiátrica o psicológica es uno de los predictores más sensibles de suicidio ${ }^{35}$. También se ha encontrado que en repetidas investigaciones que han empleado la autopsia psicológica, concuerdan en el hallazgo de patologías psiquiátricas en casi la totalidad de los sujetos o que la prevalencia de trastornos mentales es similar en suicidios consumados e intentos de suicidio ${ }^{36}$. Siomopoulos (1990) en su estudio observó que el 98\% de los sujetos que se suicidan en EEUU presentaban patología psiquiátrica ${ }^{37}$. Por otro lado, Pérez Barrero (1999) considera que tanto en la niñez, adolescencia, edad adulta y tercera edad, debe tenerse como factor de riesgo principal la existencia de patología mental $^{38}$.

Los resultados de nuestra muestra son que de la muestra de sujetos suicidas que se pudo recoger cuál era su diagnóstico anterior, el mayor porcentaje tenía diagnóstico de Trastorno del estado de ánimo depresivo (24,2\%), seguidos de los Trastornos con abuso de sustancias y Esquizofrenia. En numerosos estudios se obtiene una incidencia de trastorno de ánimo depresivo entre un 57-60\% de los pacientes que cometen un acto suicida ${ }^{35}$. La tasa estandarizada de mortalidad son 30 a 40 veces mayores de los esperados para la población general. En el caso de pacientes diagnosticados de esquizofrenia, las tasas de suicidio fluctúan entre el 18 y el 55,1\%, la ideación suicida se presenta en el $12-47 \%$ y alrededor del 10\% consuma el suicidio. Por último, en personas con trastorno por abuso de alcohol y otras sustancias, el riesgo vital de muerte por suicidio es 60-120 veces mayor que en población general. Montalvo et al. (2009) en su estudio encontró que en el Eje I el diagnóstico más prevalente fue la depresión unipolar tanto en las tentativas como en suicidios consumados ${ }^{20}$; Gradillas (1998), considera que la correlación entre el suicidio y la depresión es muy alta, siendo la propia sintomatología (tristeza, baja autoestima, pesimismo, culpabilidad, etc.) lleva a la pérdida del sentido de la vida y a la desesperanza ${ }^{39}$; Pérez Barrero (1999) considera que el alcoholismo es un de los factores de riesgo que no se puede olvidar ya que las propias características de la enfermedad (bajo estado de ánimo, aislamiento, problemas económicos, etc.) van a propiciar la autodestrucción entre los alcohólicos ${ }^{38}$. En esta línea, mencionar a Karl Menninger, el cuál propuso el término suicidio crónico para aquellos que escogen autodestruirse por medio del consumo de alcohol, drogas o de otros métodos colaterales.

A su vez, se obtiene que un $44,7 \%$ de estos pacientes, estaban en tratamiento en la actualidad y de estos, el 74\% recibían tratamiento psiquiátrico y/o psicológico. Existe una carencia de datos respecto a si el paciente estaba en tratamiento o no en un $31,9 \%$. Sin embargo, coincide los datos aportados en el estudio de Ayuso (2009), en cuya muestra obtiene que un $68,9 \%$ de los sujetos con tentativa suicida presentaba antecedentes de atención psiquiátrica previa en Centros de Salud Mental principalmente y en un 32,8\% eran pacientes con una enfermedad de evolución de más de 10 años ${ }^{11}$.

Respecto al factor desencadenante de la conducta suicida, los resultados aportan que en el mayor porcentaje $(43,55 \%)$ identifica como factor desencadenante la propia enfermedad física o psíquica. Esto puede venir relacionado con el mayor porcentaje de sujetos que presentaban patología psiquiátrica en la muestra estudiada y que su propia sintomatología provoca la conducta suicida. La OMS (2002) propone como primera medida de prevención de la conducta suicida el tratamiento de las enfermedades mentales ${ }^{2}$. Existen otros autores que proponen como principales desencadenantes o eventos o vivencias que ocurren inmediatamente antes que la conducta suicida, pero que por sí solos no son la causa de la misma («son la gota que colma el vaso»), los problemas familiares y /o de pareja, problemas legales, económicos y escolares. La influencia del estrés y los acontecimientos vitales sobre la conducta suicida han sido objeto de amplia investigación. Estudios confirman una elevada incidencia de acontecimientos vitales estresantes de tipo negativo en los meses anteriores al acto suicida ${ }^{40,41}$. Durante los seis meses previos a la realización de una tentativa, los acontecimientos estresantes son 4 veces más frecuentes entre los sujetos que llevan a cabo la tentativa que respecto a la población general, y además, una tercera parte de estos acontecimientos ocurren durante el mes anterior a la tentati$\mathrm{va}^{40}$. El suicidio puede desencadenarse por una sucesión de de acontecimientos vitales estresantes actuando durante periodos de tiempo más o menos prolongados, lo cuál se ha llamado "carrera suicida». Según Ros Montalban (1998), tanto los estresores puntuales como aquellos que se mantienen más en el tiempo, pueden ser los catalizadores que desencadenen la conducta suicida pero no está claro si son específicos para el suicidio o bien, actúan a través de la producción de psicopatología.

\section{CONCLUSIONES}

- A través de los datos aportados, podemos concluir que existe un perfil establecido de paciente suicida atendido en el ámbito extrahospitalario, el cuál permite conocer las características de lo que es en muchas ocasiones la primera demanda de asistencia o petición de ayuda.

- Se establece un perfil de paciente suicida como varón, de 2535 años, español, soltero, con patología psiquiátrica en tratamiento, sin tentativas previas, que había verbalizado sus intenciones autolíticas, teniendo como factor desencadenante la propia enfermedad física o psíquica, que elige como método autolítico la defenestración sin presencia de otras personas, al cuál se le traslada al Hospital y presenta como diagnóstico principal TCE, trauma o politraumatismo.

- Debido a que existe un porcentaje alto de sujetos con conducta suicida que previamente han tenido tentativas previas, verbalizado sus intenciones y /o que reciben tratamiento psiquiátrico/ psicológico, se considera necesario que se pongan en marcha actuaciones para una mayor detección y prevención de estos actos.

- Se considera necesario analizar en investigaciones futuras el efecto que produce la conducta suicida en el entorno. 


\section{Emergencias extrahospitalarias: el paciente suicida}

\section{BIBLIOGRAFÍA}

1. Organización Mundial de la Salud, (2002). Informe mundial sobre violencia y salud: resumen, 23-25.

2. Organización Mundial de la Salud, (2002). The World Health Report 2002. Reducing risks, promoting healthy life. Geneva: World Health Organization.

3. Ruiz-Pérez, I., Orly, A. (2006). El suicidio en la España de hoy. Gac Sanit, Mar 20(1)

4. Bernal, M., Haro, J. M., Bernert, S., Brugha, T., de, G. R., Bruffaerts, R. et al. (2007). Risk factors for suicidality in Europe: results from the ESEMED study.J.Affect.Disord., 101, 27-34.

5. Gabilondo, A.,. Alonso, J., Pinto-Meza, A., Vilagut, G., Fernández, A., Serrano Blanco, A., Almansa, J.,Codony, M., Haro, J.M. (2007). Prevalencia y factores de riesgo de las ideas, planes e intentos de suicidio en la población general española: Resultados del estudio ESEMeD Medicina clínica, Vol. 129, №. 13, 2007 , págs. 494-500.

6. Bertolote, J.M., Fleischmann, A. (2002). A global perspective in the epidemiology of suicide. Suicidology; 7. 6 - 8.

7. Philips, M.R., Li Zhang, Y. (2002). Suicide rates in China 1995-99.Lancet, 359: 835-40.

8. Instituto Nacional de Estadística (2007). Estadística del Suicidio en España en el 2006, INE.

9. Mingote, J.C; Jiménez, M.A; Osorio, R; Palomo, T. (2004). Suicidio. Asistencia Clínica. Guía de práctica médica. Ed. Díaz Santos. Cap. 4, p.19-30.

10. Mateos, A.A., Huerta, M., Benito, M.A. (2007). Características epidemiológicas del intento autolítico por fármacos. Emergencias; 19:251-254.

11. Ayuso, J.L. (2009). Estudio de la conducta autolítica en la Comunidad de Madrid: informe de resultados 2008. Departamento de Psiquiatría de UAM. Madrid.

12. Durkheim, E. (1992). El suicidio, $3^{\mathrm{a}}$ ed.,Ed. Akal, Fuenlabrada, Madrid.

13. Pacheco, T. (2008). Intento autolítico mediante ingesta de fármacos y tóxicos: perfil psicosocial en un servicio de emergencia prehospitalaria. Artículo presentado en Seminario de Drogadicción del Doctorado de Psicología Clínica, Legal y Forense (pendiente de publicación).

14. Torres, F., Almagro, V., Vargas, M.I., Rodríguez, C. y Canencia, C. (2005). «192 pacientes precipitados atendidos por un Servicio de Emergencias Prehospitalarias». Póster presentado en XVI Congreso Nacional Sociedad Española de Medicina de Urgencias y Emergencias. Palma de Mallorca.

15. Sarró, B. y De la Cruz, C. (1991). Los suicidios. Ediciones Martínez Roca. Barcelona.

16. Diekstra, R. (1993). The epidemiology of suicide and parasuicide. Acta Psychiatr Scand; 371, 9-20.

17. Diekstra, R. Y Gulbinat, W. (1993). The epidemiology of suicidal behavior: a review of three continents. Wld hlth statist quart; 46,52 - 68 .

18. Organización Mundial de la Salud (1995). 1994 World health statistics annual. Geneva.

19. Hintikka, J., Pesonen, T., Saarinen, P., Tanskanen, A., Lehtonen, J., \& Viinamaki, H. (2001). Suicidal ideation in the Finnish general population. A 12-month followup study. Soc.Psychiatry Psychiatr.Epidemiol., 36, 590 - 594.

20. Montalvo, I., Parra, I., Gisbert, L. (2009). Diferencias entre intentar y consumar el suicidio. Revista de Psiquiatria y Salud Mental,oct (2).

21. Bobes, J., Sáiz, P.A., García- Portilla, M.P., Bascarán, M.T., Bousoño, M. (2004) Comportamientos Suicidas. Prevención y Tratamiento. Ars Médica. Barcelona.
22. Quintanilla, M.A.; Villas, E.; Mabry, S; Calvo, D.; Gracia-García, P.; Argemí, M; Casanova, N; Latorre, J.I.; García-Gómez, M.A. (2008). Conductas suicidas en población inmigrante: un enfoque cultural. (Accedido 10 Enero 2009). Disponible en www.psicoadolescencia.com.ar

23. Davidson, F., Philippe, A., (1986). Suicide et tentatives de suicides aujourd'hui. Étude epidemiologique. Paris : INSERM/Doin.

24. Pacheco, T. (2008). Revisión del perfil español y sentencias en la jurisprudencia española en conductas suicidas. Trabajo presentado en Seminario de Psicopatología del Doctorado de Psicología Clínica, Legal y Forense (trabajo no publicado).

25. Rodríguez, R., Pedraza, M., Burunate, M. (2004). Factores predisponentes y precipitantes en pacientes atendidos por conducta suicida. Rev Cub Med Mil; 33 (1)

26. Harris, R., (1981). Pathways to suicide: a survey of self destructive behaviours. Baltimore: John Hopkins University Press.

27. Martí, G. y Corbella, J. (1988). Evolución del suicidio consumado en Barcelona. Jano, 2 (9), $27-31$.

28. Aso, J.; Navarro, J.A. y Cobo, J.A. (1993). El suicidio consumado en Zaragoza (1988 -1991). Aspectos epidemiológicos y perfiles suicidas. Actas Luso-Esp Neurol Psiquiatr; 21, (3), 65-71.

29. Sarró, B. y Nogué, S. ( 1992). Suicidios. Med Clín (Barc); 98, (16), 624-626.

30. Ros, S y Rodríguez, R. (1993). Estudio epidemiológico del suicidio en Barcelona en el año 1992. Ponencia presentada en las II Jornadas de la Sociedad Española de Psiquiatría Legal. Madrid.

31. Organización Mundial de la Salud, (2008). Métodos de suicidio: comparación internacional a partir de la base de datos de mortalidad. Boletín OMS; 86 (9), 726-732.

32. Buzan, R.D., Weissberg, M.P. (1992). Suicide: risk factors and prevention in medical practice. Annu Rev Med; 43, 37-46.

33. King, E. (1994). Suicide in the mentally ill. An epidemiological sample and implications for clinicians. British Journal of Psychiatry; 165, 658-663.

34. Nieto, E., Vieta, E. y Cirera, E. (1992). Intentos de suicidio en pacientes con enfermedad orgánica. Med Clin, 98: 618-621. Barcelona.

35. Torrico, E., Remesal, R., Andrés, M. (2009). El suicidio en la edad adulta. Consejo General de Colegios Oficiales de Psicólogos. Séptima edición Focad.

36. Beautrais, A.L., Joyce, P.R., Mulder, R.T. , Fergusson, D.M., Deavoll, B.J., Nightingale, S.K. (1996). Prevalence and comorbidity of mental disorders in persons making serious suicide attempts: a cas-control study. Am J Psychiatry; Aug, 153(8): 1009-14.

37. Siomopoulos V. (1990) When patients consider suicide? Risk factors to watch for Postgrad Med 1990;88(3):205-13.

38. Pérez Barrero, S. (1999). El suicidio, comportamiento y prevención. Rev Cubana Med Gen Integr; 15 (2): 196-217.

39. Gradillas, V. (1998). Psicopatología Descriptiva. Signos, síntomas y rasgos. 265 276. Pirámide

40. Adam, K.S. (1990). Environmental psicosocial and psychoanalitic aspects of suicdal behavior. En: Blumenthal, S.J. and Kupfer, D.J. Suicide over the life cycle. American Psychiatric Press, Inc. Washington.

41. Yufit, R.L. y Bongar, B. (1992). Suicide, stress and doping with life cycle events. En: Marris, R.W., Berman, A.L., Maltsberger, J.T. and Yufit, R.I. Assessment and prediction of suicide. Guilford Press. New York. 